\title{
Repulsive guidance molecules, novel bone morphogenetic protein co-receptors, are key regulators of the growth and aggressiveness of prostate cancer cells
}

\author{
JIN LI, LIN YE, HOWARD G. KYNASTON and WEN G. JIANG
}

Metastasis and Angiogenesis Research Group, Cardiff University School of Medicine, Heath Park, Cardiff, CF14 4XN, UK

Received August 4, 2011; Accepted September 29, 2011

DOI: $10.3892 /$ ijo.2011.1251

\begin{abstract}
Repulsive guidance molecule (RGM) family members RGMA, RGMB and RGMC are GPI-linked membrane proteins recently identified as co-receptor of bone morphogenetic proteins (BMPs). BMPs are a group of proteins enriched in bone and play important roles in prostate cancer. The current study aimed to investigate roles played by RGMs in prostate cancer. Expression of RGMs was examined in prostate cancer cell lines and prostate cancer tissues using RT-PCR and immunohistochemical staining. Knockdown of each RGM in prostate cancer cells was performed using the respective anti-RGMA, RGMB and RGMC transgenes. A variety of in vitro function tests were employed to analyze the influence on cancer cell functions by RGM knockdown. The implications of RGM knockdown in BMP signalling were also examined using both Western blot and real-time quantitative PCR . Knockdown of RGMA had no effect on cell growth, migration and invasion, but promoted cell-matrix adhesion. Knockdown of RGMB and RGMC increased growth and adhesion, but only RGMB knockdown increased capacities of migration and invasion in PC-3 cells. Further investigations showed an increase in Smad-3 activation and reduced levels of Smad-1 in PC-3 cells by RGMB and RGMC knockdown, and also an up-regulation of ID1, a BMP target gene particularly in exposure to BMP7. RGMs play inhibitory roles in prostate cancer by suppressing cell growth, adhesion, migration and invasion. RGMs can coordinate Smad-dependent signalling of BMPs in prostate cancer cells.
\end{abstract}

\section{Introduction}

Bone morphogenetic proteins (BMPs) have been indicated in the disease progression of prostate cancer, particularly in the

Correspondence to: Professor Wen G. Jiang, Metastasis and Angiogenesis Research Group, Department of Surgery, Cardiff University School of Medicine, Heath Park, Cardiff, CF14 4XN, UK

E-mail: jiangw@cf.ac.uk

Key words: repulsive guidance molecule, bone morphogenetic protein, prostate cancer, Smad, ID1 disease specific bone metastasis (1). A group of novel BMP co-receptors, namely repulsive guidance molecules (RGMs)-A, $-B$ and $-\mathrm{C}$ were recently identified. RGMs are GPI-linked membrane proteins, sharing 50-60\% sequence homology (2-4). RGMA mediates repulsive axonal guidance and neural tube closure, and RGMC is mutated in juvenile hemochromatosis. RGMB, also known as Dragon, is a myelin-derived inhibitor of axon growth in the central nerve system (5) and regulates the patterning of the developing nervous system (6). As BMP co-receptors, RGMA can increase binding of BMP ligands to ActRIIA, a type II receptor, and lead to an enhanced signalling by BMP-2 and BMP-4 (7). RGMA enhances signalling of BMP-2 and BMP-4, leading to activation of Smad dependent pathway and up-regulation of a target gene, ID1 (2). RGMA is also crucial as a co-receptor for Hepcidin expression induced by BMP-2 and BMP-4, thus to maintain systemic iron homeostasis (8). RGMB directly interacts with BMP receptors, enhancing binding to their ligands, such as BMP-2 and BMP-4, but not BMP-7 (6). RGMB can negatively regulate IL-6 expression in immune cells through Smad-independent signalling by BMPs, including p38 and ERK pathways (9). On the other hand, upon binding to its ligand neogenin, RGMA may also mediate growth cone collapse in neurites which has been shown as independent from BMPs signalling (10). A recent study also revealed that secreted von Willbrand factor type D domain of RGMB suppressed Smad-dependent signal transduction by BMPs, in which unknown receptors were involved (11). Similarly, upon binding to its receptor neogenin, RGMC is stablized by anchoring on the cell membrane, which could compromise its inhibition on BMP signalling (12).

BMPs play profound roles in prostate cancer and the disease specific bone metastasis by regulating proliferation, apoptosis and motility of prostate cancer cells, and also tumour associated angiogenesis. For example, BMP-7 and BMP-9 inhibit the proliferation of $\mathrm{PC}-3$ prostate cancer cells via inducing apoptosis through BMP receptors and Smad-dependent pathway $(13,14)$, while BMP-10 inhibits the growth of prostate cancer cells via Smad-independent signalling in which XIAP and ERK1/2 are involved (15). Therefore as BMP co-receptors, RGMs might also be involved in the prostate cancer development and metastasis through the participation in BMP signalling pathway. To date, there is only one study investigated the implication of RGMs in cancer, in which an epigenetic down-regulation of 
RGMA was revealed in colorectal carcinomas, adenomas and colorectal cancer cell lines (16). However, the role played by RGMs in prostate cancer remains unknown. The present study aimed to examine the expression of RGMs in prostate cancer and their functions in prostate cancer cells.

\section{Materials and methods}

Materials. Antibodies to RGMA, RGMB, RGMC, phosphorylated Smad-1, and Smad-3 were obtained from Santa Cruz Biotechnology, CA. Human recombinant protein BMP-7 (rh-BMP7) was purchased from R\&D System, Inc. (Abingdon, UK). All the primers were synthesized by Invitrogen (Paisley, UK), as shown in Table I.

PC-3 cell line was purchased from European Collection of Animal Cell Culture, Salisbury, UK; DU-145, LNCaP, CA-HPV-10 and PZ-HPV-7 from American Type Culture Collection. PNT-1A and PNT-2C2 were kindly provided by Professor Norman Maitland (University of York, UK). The cells were routinely maintained in DMEM supplemented with $10 \%$ fetal calf serum and antibiotics.

Prostate tissue samples were snap-frozen in liquid nitrogen immediately after radical prostatectomy, transurethral prostatectomy or prostate biopsy. All protocols were reviewed and approved by the local ethics committee and all patients gave written informed consent.

Immunohistochemical (IHC) staining procedure for frozen prostate tissue. Frozen specimens of prostate tumours $(\mathrm{n}=8)$ and normal prostate tissues $(n=13)$ were cut at a thickness of $6 \mu \mathrm{m}$ using a cryostat, and verified by two pathologists. IHC was performed using respective primary antibody and Vectastain Universal Elite ABC kit (Vector Laboratories, Peterborough, UK). The density of the IHC staining was quantified using ImageJ (http://rsbweb.nih.gov/).

Generation of RGM ribozyme transgenes. Specific hammer head ribozymes targeting human RGMA, RGMB and RGMC were designed and synthesised following previously described procedure (17). The ribozymes were cloned into $\mathrm{pEF} 6 / \mathrm{His}$ TOPO mammalian expression plasmid vectors (Invitrogen Inc., Paisley, UK). Control empty plasmid vectors and the ribozyme transgenes were then transfected into PC-3 cells, followed by two weeks of selection using $5 \mu \mathrm{g} / \mathrm{ml}$ blasticidin. Knockdown of specific RGM was then confirmed before further use.

RNA isolation, reverse transcription and polymerase chain reaction $(P C R)$. RNA isolation was carried out using TRI reagent from Sigma. RNA $(0.5 \mu \mathrm{g})$ was converted into cDNA using the iScript ${ }^{\mathrm{TM}}$ cDNA Synthesis kit (Bio-Rad, Hemel Hemstead, UK).

Real-time quantitative PCR. The real-time quantitative PCR was carried out to determine the levels of RGM transcripts and ID1 in the control and RGM knockdown cells. The assay was based on the Amplifuor technology and primers were designed using Beacon Designer software which included complementary sequence to universal $\mathrm{Z}$ probe (Intergen Inc., Oxford, UK), as previously reported $(18,19)$. The primers used for RGMs and ID1 quantification and housekeeping GAPDH are listed in Table I.

Protein extraction, SDS-PAGE and Western blot analysis. Following cell lysis, the protein concentration was quantified using the DC Protein Assay kit (Bio-Rad, USA) and a spectrophotometer (Bio-Tek, ELx800). Equal amounts of protein from each sample were loaded onto $8 \%$ polyacrylamide gel and following SDS-PAGE, the proteins were transferred onto nitrocellulose membranes subjected to blocking, and probing with the specific primary (1:200), and the corresponding peroxidiseconjugated secondary antibodies (1:2000). The protein bands were eventually visualized using the Supersignal ${ }^{\mathrm{TM}}$ West Dura system (Pierce Biotechnology, USA).

In vitro cell growth assay. The cells were seeded into a 96-well plate at a concentration of 3,000 cells per well, and incubated for periods of up to 5 days. The cells were then stained with crystal violet, and measured the absorbance. The results are shown as growth rate which was calculated by normalization against the absorbance measurements at day 0 .

In vitro cell-matrix adhesion assay. 45,000 cells were seeded onto the 96-well plate coated with Matrigel basement membrane in $200 \mu \mathrm{l}$ of normal medium and incubated for $40 \mathrm{~min}$. After washing, adherent cells were fixed and visualized under the microscope under $\mathrm{x} 40$ objective magnification and random fields counted.

In vitro cell invasion assay. Transwell inserts (upper chamber) with an $8 \mu \mathrm{m}$ pore size were coated with $50 \mu \mathrm{g}$ /insert of Matrigel and air-dried, before being rehydrated with normal medium. Then, $2 \times 10^{4}$ cells were added to each well. After $72 \mathrm{~h}$, cells that had migrated to the other side of the insert through the matrix were fixed and stained with $0.5 \%(\mathrm{w} / \mathrm{v})$ crystal violet. The invaded cells stained with crystal violet were counted under a microscope.

In vitro cell motility assay using cytodex-2 beads. Cellular motility was assessed using a cytodex-2 bead motility assay. Cells $\left(5 \times 10^{5}\right)$ for each cell type were incubated in $10 \mathrm{ml}$ of growth medium containing $100 \mu \mathrm{l}$ of cytodex- 2 microcarrier beads (GE Healthcare, Cardiff, UK) for $3.5 \mathrm{~h}$ to allow the cells to adhere to the beads. After washing the beads were resuspended in growth medium, seeded into a 24 -well plate and then incubated overnight at $37^{\circ} \mathrm{C}$. Cells that had migrated from the beads and adhered to the base of the well were fixed in $4 \%$ formaldehyde $(\mathrm{v} / \mathrm{v})$, stained with $0.5 \%$ crystal violet (w/v) and counted under $\mathrm{x} 40$ objective magnification.

ECIS-based attachment and migration assay. We used the electric cell-substrate impedance sensing (ECIS) system (Applied Biophysics, Inc., Troy, NY) to conduct the attachment and wounding assay). PC-3 cells were cultured in 8W1E ECIS arrays (Applied Biophysics). We seeded PC-3 cells at a density of 70,000 cells/well in the arrays. The cells were incubated for $4 \mathrm{~h}$ and the resistance was recorded every $15 \mathrm{sec}$. Once the cells fully attached, the monolayer was electrically wounded by applying an elevated voltage pulse with a frequency of $30 \mathrm{kHz}$, 
Table I. Primers used in this study.

\begin{tabular}{lll}
\hline Gene & \multicolumn{1}{c}{ Sense (5'-3') } & \multicolumn{1}{c}{ Anti-sense (5'-3') } \\
\hline RGMA & TCGACAATAATTACCTGAACG & ACTGAACCTGACCGTACACCTGGAAGTTCTTGAAGATG \\
RGMB & TTCAGGTTCAAGTGACAAACG & ACTGAACCTGACCGTACATCATCTGTCACAGCTTGGTA \\
RGMC & AATGACTTCCTCTTTGTCCA & ACTGAACCTGACCGTACACATTCCTGCATGTTCTTAAA \\
RGMA & CTGCAGGCGCACCACGATGGTGGTGCCC & ACTAGTGGAGATCCAGGCCAAGTACATTTCGT \\
ribozyme & TGATGAGTCCGTGAGGA & CCTCACGGACT \\
RGMB & CTGCAGGTCATCTGTCACAGCTTGGTA & ACTAGTGTACAGATCAGAAAGTTTCGTCCTCACGGACT \\
ribozyme & CTGATGAGTCCGTGAGGA & \\
RGMC & CTGCAGATCCCCCAGGTCGGTCACCTCC & ACTAGTCCTGTAGCCTTTGAAGATGGTTCTATTTCGTC \\
ribozyme & ATTCTGATGAGTCCGTGAGGA & CTCACGGACT \\
ID1 & TCAACGGCGAGATCAG & ACTGAACCTGACCGTACAGATCGTCCGCAGGAA \\
GAPDH & AAGGTCATCCATGACAACTT & ACTGAACCTGACCGTACAGCCATCCACAGTCTTCTG \\
\hline
\end{tabular}

amplitude of $4 \mathrm{~V}$ for $10 \mathrm{sec}$. Cell migration was assessed by continuous resistance measurements for $30 \mathrm{~h}$.

Statistical analysis. Normally distributed data were analyzed using the two sample t-test while non-normally distributed data were analyzed using the Mann-Whitney and Kruskal-Wallis tests.

\section{Results}

Expression of RGMs in prostate cancer. The expression of RGMs was examined in prostate cancer cell lines and prostate cancer tissues using RT-PCR. RGMs were expressed in most of the prostate cancer cell lines. No remarked difference was seen in RGM expression between prostate cancer cells and the immortalised prostatic epithelial cells. RGMB was not expressed in CAHPV-10 cells, and RGMC was barely detectable in LNCaP cells. Among the RGMs, RGMA mRNA was hardly detectable in the two prostate cancer tissues examined (Fig. 1).

To further investigate the expression of RGMs in prostate tissues, we examined their protein levels using immunohistochemical staining. RGM protein was mainly confined to the cytoplasm of cancer cells and some of the mesenchymal cells on the periphery of the tumour area. The immunochemical staining of RGMB and RGMC protein revealed subtle stronger staining in prostate cancer cells compared to prostate epithelial cells, while staining of RGMA is much weaker in both prostatic epithelia and cancer cells (Fig. 2A). The intensity of the staining in the cells was measured and calculated based on the background using ImageJ. The quantification of the IHC staining shows the levels of RGMB and RGMC in prostate tumours were significantly higher, compared to background tissues $(\mathrm{P}<0.05)$ (Fig. 2D).

Knockdown of RGMA, RGMB and RGMC in PC-3 cells. To test the potential role of RGMs in prostate cancer cells, knockdown of RGMA, RGMB and RGMC in PC-3 cells were performed using the respective anti-RGM transgenes. The knockdown of

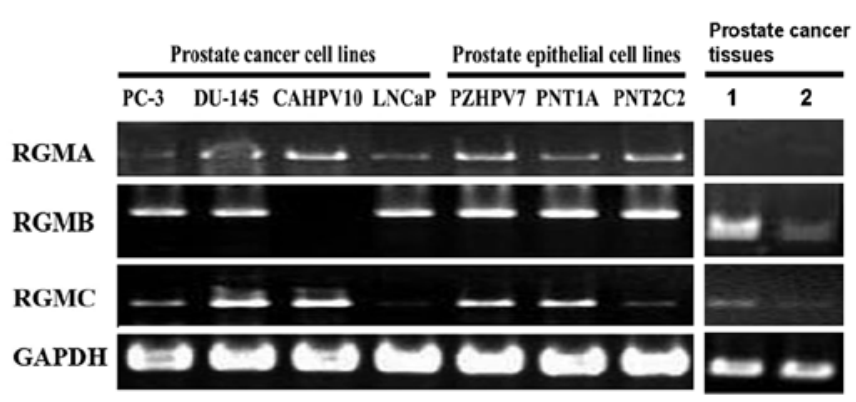

Figure 1. RT-PCR shows expression pattern of RGMs in prostate cancer cell lines. RGMs were expressed in most the prostate cancer cell lines and the immortalized prostatic epithelial cell lines. In the two prostate cancer tissue samples examined in current study, RMGB was expressed at relatively higher levels.

RGMs mRNA and protein were then verified using both realtime quantitative PCR and Western blotting (Fig. 3).

Effect on in vitro growth of prostate cancer cells by RGMs knockdown. Following the verification of knockdown, we examined effect on functions of prostate cancer cell by RGMs knockdown. Firstly, we assessed the influence on cell growth. Compared to control cells, PC-3 $3^{\triangle \mathrm{RGMA}}$ cells did not show difference in cell growth $(\mathrm{p}=0.07)$. In contrast, PC- $3^{\triangle \mathrm{RGMB}}$ and PC- $3^{\triangle \mathrm{RGMC}}$ cells showed increase in cell growth, both $\mathrm{p}<0.05$ compared to $\mathrm{PC}-3^{\mathrm{pEF}}$ control cells (Fig. 4A).

Influence on cell-matrix adhesion by knockdown of RGMs in prostate cancer cells. In the adhesion assay, reduced expression of RGMs (RGMA, B and C) in PC-3 cells increased cell adhesion, compared to the control cells. The number of cells adhered to matrix for PC-3 $3^{\triangle \mathrm{RGMA}}(26.93 \pm 13.23), \mathrm{PC}-3^{\triangle \mathrm{RGMB}}(30.67 \pm 12.43)$, PC- $3^{\triangle \mathrm{RGMC}}(30 \pm 12.43)$ were increased, $\mathrm{p}<0.05$ compared to PC $-3^{\mathrm{pEF}}$ control cells (16.67 \pm 8.09 ) (Fig. 4B).

Effect on motility and invasiveness of prostate cancer cells by RGMs knockdown. Furthermore, RGM knockdown cells 
A RGMA

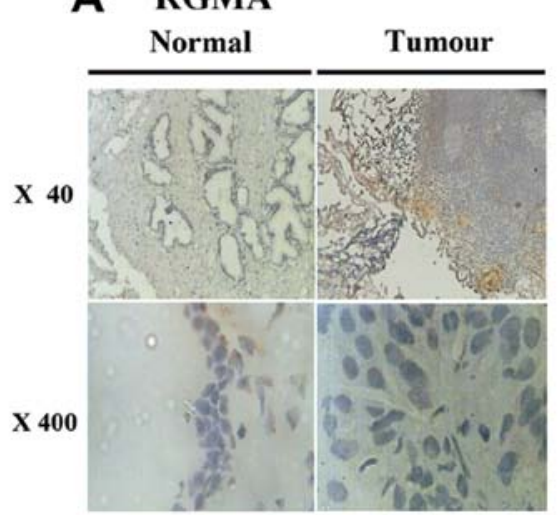

B RGMB

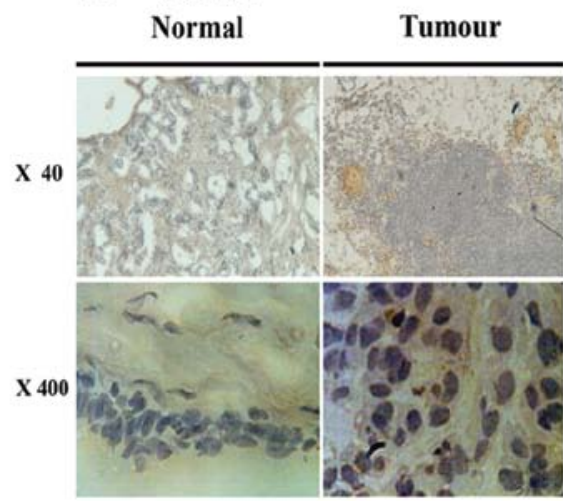

Negative control
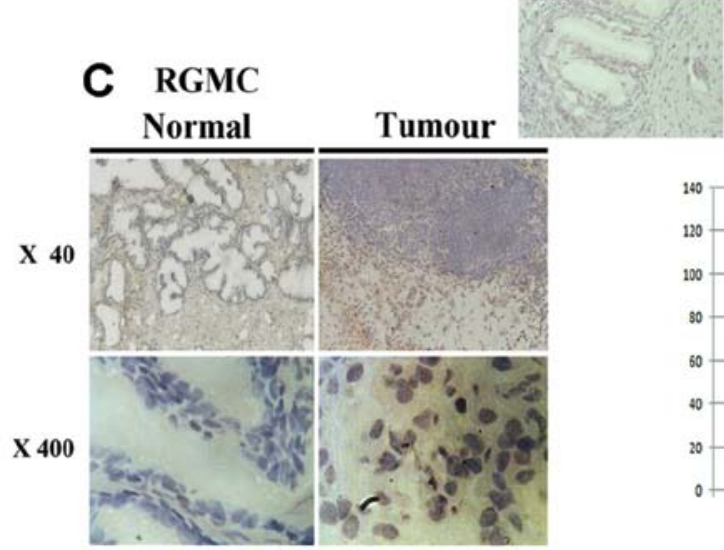

D

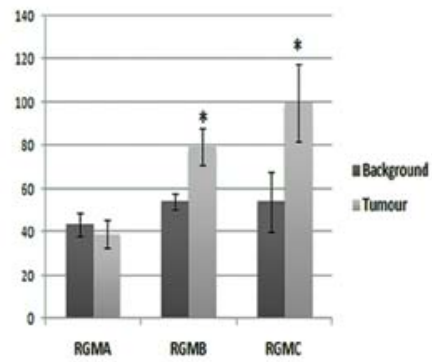

Figure 2. IHC staining of RGMs in human prostate cancer. (A-C) Immunochemical staining of RGMA, RGMB and RGMC in prostate cancer specimens, reduced from $x 40$ and $x 400$, respectively. (D) There is no significant difference of the staining of RGMA, RGMB and RGMC seen in the prostate cancer tissues when compared with background tissues using semi-quantification of the staining using ImageJ software. For negative control only secondary antibody was applied during the probing procedure. ${ }^{*} \mathrm{P}<0.05$ vs normal prostate tissue.

A

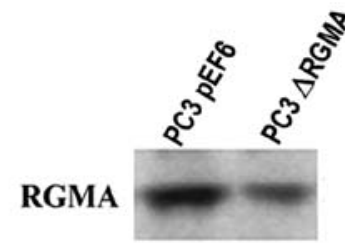

GAPDH

B

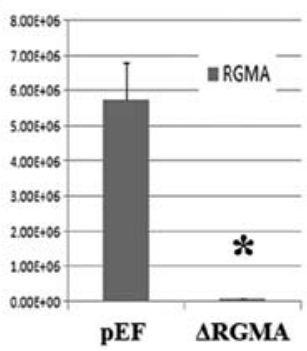

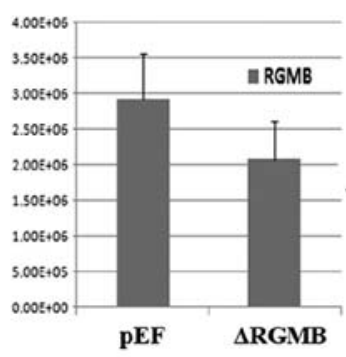

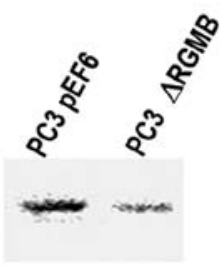

RGMB

RGMC

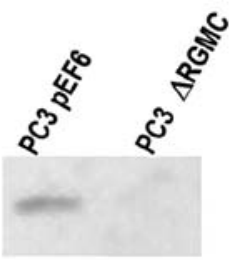

GAPDH

Figure 3. Knockdown of RGMs in PC-3 cells. (A) Reduced protein expression of RGMs was verified in PC-3 $3^{\triangle R G M A}$, PC- $3^{\triangle R G M B}$ and PC- $3^{\triangle R G M C}$ cells, in comparison with PC- $3^{\mathrm{pEF}}$ cells using Western blotting. (B) Real-time quantitative PCR revealed reduced transcript level of RGMs by ribozyme transgenes. A significant reduction of RGMA transcripts was seen in PC- $3^{\mathrm{ARGMA}}$ cells $\left(1.05 \times 10^{5} \pm 1.20 \times 10^{4}\right)$, $\mathrm{p}=0.007$ compared with PC- $3^{\mathrm{PEF}}$ control cells $\left(5.76 \times 10^{6} \pm 1.05 \times 10^{6}\right)$. Although reduced levels of RGMB and RGMC transcripts were seen in the corresponding cells, but there was no significant difference according the statistical results. " $\mathrm{P}<0.05$ vs control. 

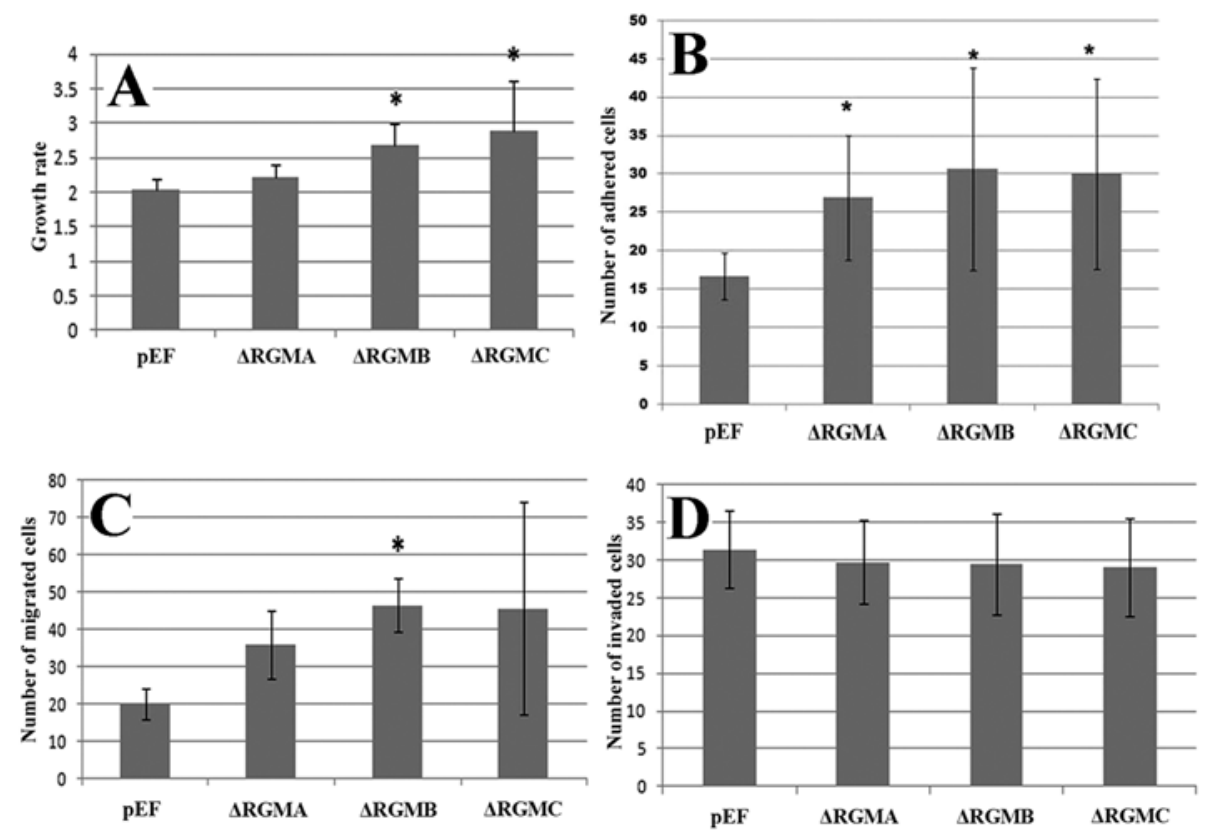

Figure 4. Effects on in vitro functions of PC-3 cells by RGMs knockdown. (A) Shown is the absorbance of day 3 which represented the cells number of each cell lines. The reduction of RGMB and C in PC-3 significantly promoted growth rate. (B) Knockdown of RGMs promoted cell-matrix adhesion of prostate cancer cells. (C) Knockdown of RGMB and RGMC resulted in elevated motility in PC-3 cells. (D) The invasiveness of PC-3 cells had not been altered by knockdown of RGMs. Minimum three independent experiments were conducted and a representative example is shown. ${ }^{~} \mathrm{P}<0.05 \mathrm{vs} \mathrm{PC}-3^{\mathrm{pEF} / \mathrm{His}}$.
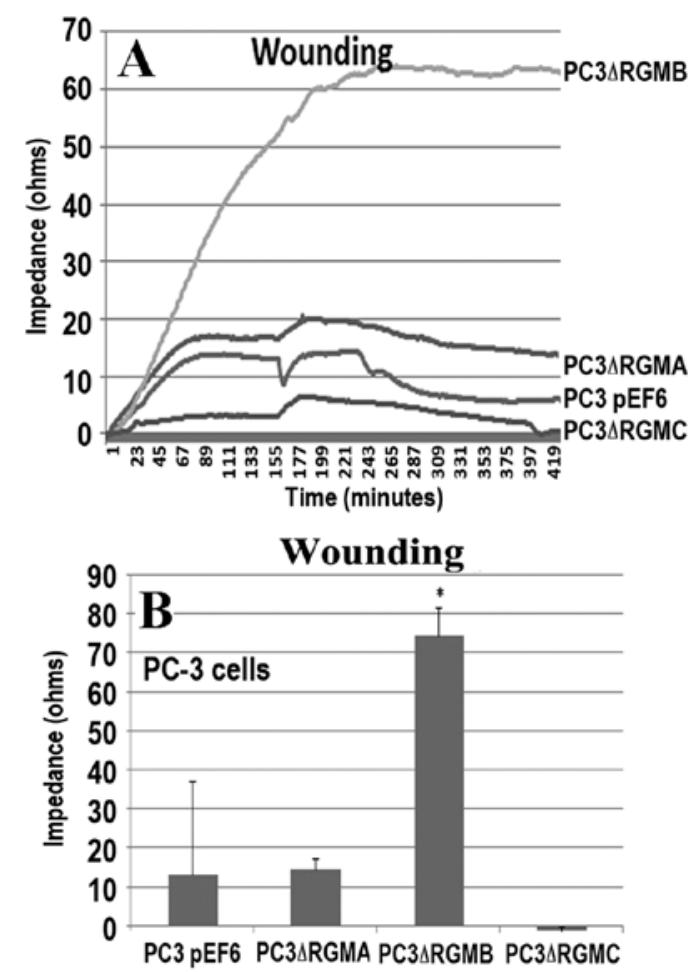

Figure 5. Effect on cell migration by knockdown of RGMs was also determined using the ECIS assay. (A) PC-3 ${ }^{\triangle R G M B}$ cells showed marked increase in migration after wounding. (B) The mean of increased impedance (ohms) at $1 \mathrm{~h}$ after wounding was calculated based on three individual experiments. Error bars presents standard error. ${ }^{*} \mathrm{P}<0.05$ vs $\mathrm{PC}-3^{\mathrm{pEF} / \mathrm{His}}$.

displayed increased motility, (Fig. 4C). The number of migrated PC- $3^{\triangle \mathrm{RGMA}}$ cells was $36 \pm 9.2$, being $46.4 \pm 7.06$ and $45.54 \pm 28.46$ for PC-3 $3^{\triangle \mathrm{RGMB}}$ and PC-3 ${ }^{\triangle \mathrm{RGMC}}$ cells, respectively. However, only the increase in $\mathrm{PC}-3^{\mathrm{ARGMB}}$ cells was statistically significant $(\mathrm{p}<0.01)$ compared with PC- $3^{\mathrm{pEF}}$ control cells $(20.2 \pm 4.06)$.

To further confirm the effect on cell motility by RGMs knockdown, we examined the cell migration using ECIS. The migration rate was much higher of PC- $3^{\triangle R G M B}$ cells compared to control, while the RGMA and RGMC knockdown has no significant effect on the migration (Fig. 5A). One hour after the wounding, the average impedance in PC- $3^{\triangle \mathrm{RGMB}}$ cells was significantly higher than the $\mathrm{p}^{\mathrm{EF}}$ control cells $(\mathrm{p}=0.009)$. However, the RGMA and RGMC knockdown cells did not show such significant effect on migration in the ECIS wounding assay (Fig. 5B). This is thus consistent with the observations using the afore-mentioned motility assay. We further demonstrated that knockdown of RGMs had no effect on invasion of prostate cancer cells (Fig. 4D).

Potential roles of RGMs in BMP Smad-dependent signalling and regulation of $I D 1$ expression. To investigate effect on BMP signalling by RGMs knockdown in PC-3 cells, we analyzed levels of phosphorylated Smad-1 and Smad-3 in the cells, and also transcript levels of ID1. RGMs knockdown affected BMP signalling in PC-3 cells, the BMP downstream signalling molecules (Smads) activation profile was examined, which might be associated with ID1 expression in these transfected cells.

RGMB and RGMC knockdown in PC-3 cells significantly increased Smad-3 activation, but not in PC-3 $3^{\triangle \mathrm{RGMA}}$ cells, compared to the control cells (Fig. 6A and B). The increased activation was enhanced by stimulation of rh-BMP7 $(40 \mathrm{ng} / \mathrm{ml}$, $30 \mathrm{~min}$ ), which indicated RGMB and RGMC knockdown can enhance the Smad-3 dependent pathway activated by BMP7 (Fig. 6A and B). In contrast to the effect on Smad-3 phosphorylation, reduced levels of activated Smad-1 were seen in PC- $3^{\triangle \mathrm{RGMB}}$ and PC-3 $3^{\triangle \mathrm{RGMC}}$ cells, but not in PC-3 $3^{\triangle \mathrm{RGMA}}$ cells (Fig. 6A). 

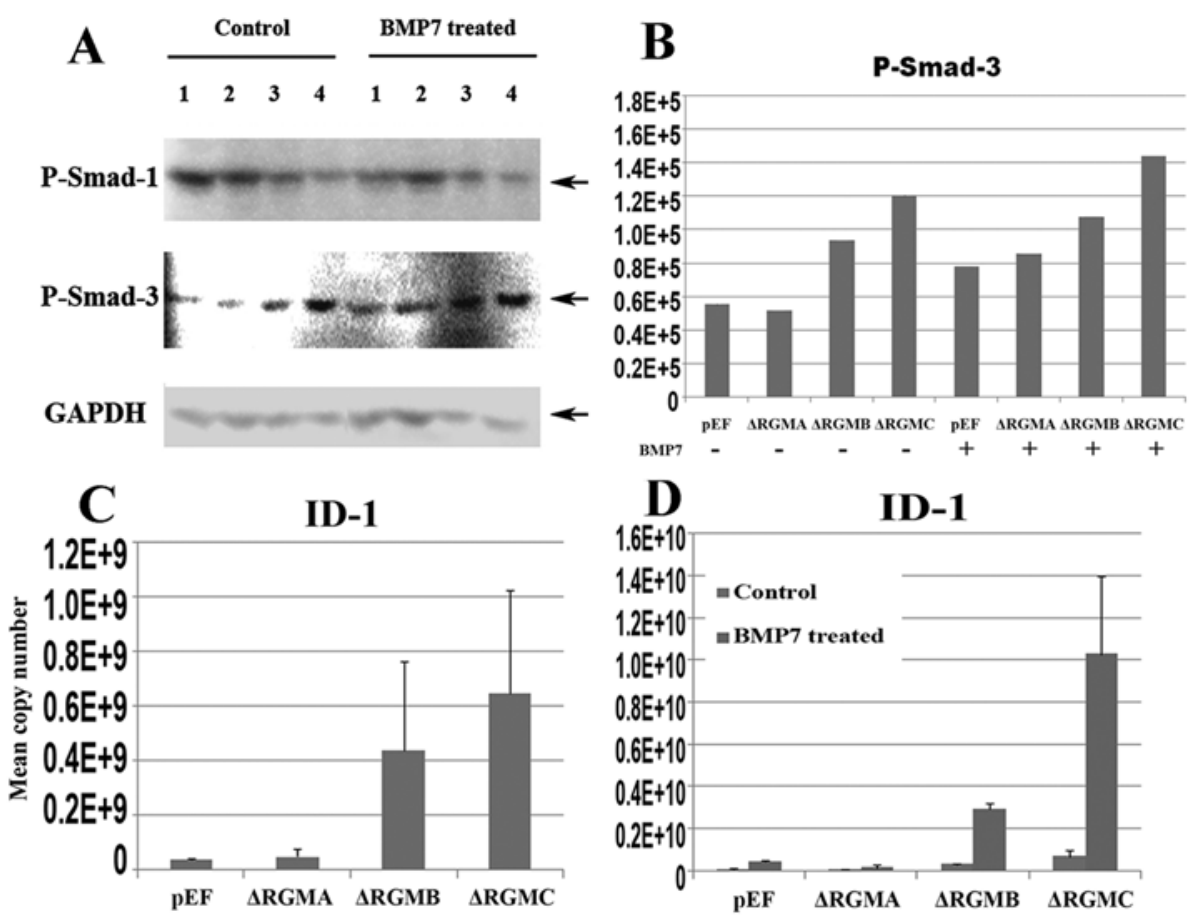

Figure 6. Influence on Smad dependent signal transduction by RGM knockdown. (A) Activated Smad-3 expression was up-regulated after RGMB and RGMC were knocked down and this is enhanced by stimulation of BMP7 (40 ng/ml, $30 \mathrm{mins}$ ), while there is no significant change in phosphorylated Smad-1 levels. (B) Shown is the band volume of P-Smad-3 being normalized against corresponding GAPDH band volume. (C) ID1 transcript levels in the transfected cells were determined using real-time quantitative PCR. ID1 transcripts in RGMA, RGMB and RGMC knockdown cells were upregulated, being $4.93 \times 10^{7} \pm 2.83 \times 10^{7}, 4.4 \times 10^{8} \pm 3.24 \times 10^{8}$ and $6.49 \times 10^{8} \pm 3.77 \times 10^{8}$ respectively), $\mathrm{p}=0.52,0.09$ and 0.05 compared to PC- $3^{\mathrm{PEF}}$ control cells $\left(3.76 \times 10^{7} \pm 0.28 \times 10^{7}\right)$. (D) The ID1 level was elevated in RGM knockdown cells in exposure to BMP7 treatment $(40 \mathrm{ng} / \mathrm{ml}, 30 \mathrm{~min}$ ) and this effect was strong in RGMB and RGMC. Shown is the copy numbers of each RGM normalised against corresponding GAPDH.

Expression of ID1, a BMP responsive gene in these cells was then determined using real-time quantitative PCR. In line with the observation of activated Smad-3 in PC- $3^{\triangle \mathrm{RGMB}}$ and PC- $3^{\triangle R G M C}$ cells, increased transcript levels of ID1 were evident in these cells and also in the cells exposed to BMP-7. Similarly, the up-regulation of ID1 was not seen in the PC- $3^{\triangle \mathrm{RGMA}}$ cells (Fig. 6C and D).

\section{Discussion}

In the present study, roles played by RGMs in prostate cancer were investigated. The expression of RGMA, RGMB and RGMC was evident in most examined prostate cancer cell lines, and also in the prostate cancer tissues. Although stronger staining of RGMB and RGMC was seen in prostate cancer cells in comparison to prostatic epithelia of normal prostate tissues, the association with disease development and progression requires further investigation in a large cohort and an animal model.

Knockdown of RGMs using hammerhead ribozyme transgenes resulted in accelerated cell growth and enhanced adhesion in vitro. Interestingly, the effects on cellular behaviour were most significant in RGMB knockdown cells. The knockdown of RGMB significantly enhanced the prostate cancer cell capacity, namely increased growth, adhesive, motility and mobility. RGMC knockdown did not affect cell motility, however, had an effect on cell growth and adhesion. In comparison to RGMB and RGMC, knockdown of RGMA only affected cell-matrix adhesion, but not growth, migration and invasion. It suggests that RGMs, particularly RGMB and RGMC trigger or mediate inhibitory effect on growth, adhesion and motility of prostate cancer cells.

To reveal what mechanism(s) is involved in the regulation of prostate cancer cells by RGMs, we investigated the effect of RGM knockdown on Smad-dependent signal transduction. An increased activation of Smad-3 was evident in the PC-3 cells by knockdown of RGMB and RGMC, while activation of Smad-1 was suppressed in these cells. It suggests RGMs, particularly RGMB and RGMC coordinate signal transduction of BMPs and divert the signal to be relayed via a different Smad.

DNA-binding protein inhibitor (ID1) proteins belong to the helix-loop-helix (HLH) transcriptional regulator family, and functions as an antagonists of basic HLH transcription factors by inhibiting their ability to bind specific DNA sequences within target gene promoters, thereby negatively regulate cell differentiation (20). Additionally, they have also been revealed to participate in tumour cell growth and promote cell survival in prostate cancer (21). ID1 is a direct downstream target gene of BMPs being induced by the BMP Smad-dependent signalling pathway $(22,23)$, promoting cell growth and inhibiting cell differentiation in breast cancer and prostate cancer $(24,25)$. In the current study, up-regulation of ID1 expression was seen in PC-3 cells by knockdown of RGMB and RGMC, which were enhanced by addition of BMP7. The increased level of ID1 in RGM knockdown cells could at least partially be responsible 
for their effects (promotion) on cell proliferation, adhesion and motility. However, the regulation of ID1 expression may be mediated via Smad-3-dependent pathway, rather than the Smad-1 pathway as previously reported (26-29). Collectively, it was indicated that RGMB and RGMC exert their regulation both on endogenous and exogenous BMPs. Interestingly, the three RGM family members in prostate cancer seem not functionally complementary and associated, as after knockdown of each one the effect will not be substituted by the other two.

In conclusion, RGMs play inhibitory roles in prostate cancer by suppressing cell growth, adhesion, migration and invasion. RGMs can coordinate Smad-dependent signalling by BMPs in prostate cancer cells. The current study indicates the interesting role of RGMs in prostate cancer and a potential therapy target in the future.

\section{Acknowledgements}

We would like to thank Cancer Research Wales and Fong Family Foundation, for their kind support.

\section{References}

1. Ye L, Mason MD and Jiang WG: Bone morphogenetic protein and bone metastasis, implication and therapeutic potential. Front Biosci 16: 865-897, 2011.

2. Babitt JL, Zhang Y, Samad TA, et al: Repulsive guidance molecule (RGMa), a DRAGON homologue, is a bone morphogenetic protein co-receptor. J Biol Chem 280: 29820-29827, 2005.

3. Papanikolaou G, Samuels ME, Ludwig EH, et al: Mutations in HFE2 cause iron overload in chromosome 1q-linked juvenile hemochromatosis. Nat Genet 36: 77-82, 2004.

4. Monnier PP, Sierra A, Macchi P, et al: RGM is a repulsive guidance molecule for retinal axons. Nature 419: 392-395, 2002.

5. Liu X, Hashimoto M, Horii H, et al: Repulsive guidance molecule $\mathrm{b}$ inhibits neurite growth and is increased after spinal cord injury. Biochem Biophys Res Commun 382: 795-800, 2009.

6. Samad TA, Rebbapragada A, Bell E, et al: DRAGON, a bone morphogenetic protein co-receptor. J Biol Chem 280: 14122-14129, 2005.

7. Xia Y, Yu PB, Sidis Y, et al: Repulsive guidance molecule RGMa alters utilization of bone morphogenetic protein (BMP) type II receptors by BMP2 and BMP4. J Biol Chem 282: 18129-18140, 2007.

8. Babitt JL, Huang FW, Wrighting DM, et al: Bone morphogenetic protein signaling by hemojuvelin regulates hepcidin expression. Nat Genet 38: 531-539, 2006.

9. Xia Y, Cortez-Retamozo V,Niederkofler V, et al: Dragon (repulsive guidance molecule B) inhibits IL-6 expression in macrophages. J Immunol 186: 1369-1376, 2010.

10. Conrad S, Genth H, Hofmann F, et al: Neogenin-RGMa signaling at the growth cone is bone morphogenetic protein-independent and involves RhoA, ROCK, and PKC. J Biol Chem 282: 16423-16433, 2007.
11. Kanomata K,Kokabu S,Nojima J,et al: DRAGON, a GPI-anchored membrane protein, inhibits BMP signaling in C2C12 myoblasts. Genes Cells 14: 695-702, 2009.

12. Lee DH, Zhou LJ, Zhou Z, et al: Neogenin inhibits HJV secretion and regulates BMP-induced hepcidin expression and iron homeostasis. Blood 115: 3136-3145, 2010.

13. Miyazaki H, Watabe T, Kitamura T, et al: BMP signals inhibit proliferation and in vivo tumor growth of androgen-insensitive prostate carcinoma cells. Oncogene 23: 9326-9335, 2004.

14. Ye L, Kynaston $\mathrm{H}$ and Jiang WG: Bone morphogenetic protein-9 induces apoptosis in prostate cancer cells, the role of prostate apoptosis response-4. Mol Cancer Res 6: 1594-1606, 2008.

15. Ye L, Kynaston $\mathrm{H}$ and Jiang WG: Bone morphogenetic protein-10 suppresses the growth and aggressiveness of prostate cancer cells through a Smad independent pathway. J Urol 181: 2749-2759, 2009.

16. Li VS, Yuen ST, Chan TL, et al: Frequent inactivation of axon guidance molecule RGMA in human colon cancer through genetic and epigenetic mechanisms. Gastroenterology 137: 176-187, 2009.

17. Jiang WG, Grimshaw D, Martin TA, et al: Reduction of stromal fibroblast-induced mammary tumor growth, by retroviral ribozyme transgenes to hepatocyte growth factor/scatter factor and its receptor, c-MET. Clin Cancer Res 9: 4274-4281, 2003.

18. Jiang WG, Davies G, Martin TA, et al: Targeting matrilysin and its impact on tumor growth in vivo: the potential implications in breast cancer therapy. Clin Cancer Res 11: 6012-6019, 2005.

19. Parr C, Watkins G and Jiang WG: The possible correlation of Notch-1 and Notch-2 with clinical outcome and tumour clinicopathological parameters in human breast cancer. Int J Mol Med 14: 779-786, 2004.

20. Norton JD: ID helix-loop-helix proteins in cell growth, differentiation and tumorigenesis. J Cell Sci 113: 3897-3905, 2000.

21. Forootan SS, Wong YC, Dodson A, et al: Increased Id-1 expression is significantly associated with poor survival of patients with prostate cancer. Hum Pathol 38: 1321-1329, 2007.

22. Miyazono K and Miyazawa K: ID: a target of BMP signaling. Sci STKE, 2002: pe 40, 2002.

23. Valdimarsdottir G, Goumans MJ, Rosendahl A, et al: Stimulation of Id1 expression by bone morphogenetic protein is sufficient and necessary for bone morphogenetic protein-induced activation of endothelial cells. Circulation 106: 2263-2270, 2002.

24. Yap WN, Chang PN, Han HY, et al: Gamma-tocotrienol suppresses prostate cancer cell proliferation and invasion through multiplesignalling pathways. Br J Cancer 99: 1832-1841, 2008.

25. Yap WN, Zaiden N, Tan YL, et al: Id1, inhibitor of differentiation, is a key protein mediating anti-tumor responses of gamma-tocotrienol in breast cancer cells. Cancer Lett 291: 187-199, 2010.

26. Ying QL, Nichols J, Chambers I, et al: BMP induction of Id proteins suppresses differentiation and sustains embryonic stem cell selfrenewal in collaboration with STAT3. Cell 115: 281-292, 2003.

27. Belletti B, Prisco M, Morrione A, et al: Regulation of Id2 gene expression by the insulin-like growth factor I receptor requires signaling by phosphatidylinositol 3-kinase. J Biol Chem 276: 13867-13874, 2001.

28. Lasorella A, Noseda M, Beyna M, et al: $\mathrm{Id} 2$ is a retinoblastoma protein target and mediates signalling by Myc oncoproteins. Nature 407: 592-598, 2000

29. Ohtani N, Zebedee Z, Huot TJ, et al: Opposing effects of Ets and Id proteins on p16INK4a expression during cellular senescence. Nature 409: 1067-1070, 2001. 